\section{Excrement analysis by PCR}

SIR - Samples from endangered animals are hard to obtain for genetic analysis. To study a threatened bear population in the Pyrenees, Taberlet and Bouvet ${ }^{1}$ used hair collected from wire netting attached to trees on which the bears scratch themselves. We have approached the problem by the use of bear droppings, which can be collected without disturbing the animals and can be used to amplify DNA sequences not only from the animals themselves but also from the foods they have ingested.

We are studying a population of European brown bears in the Brenta region of Northern Italy. Like the Pyrenean bears, this population is in decline and today may number less than 10 individuals. The introduction of animals from the large populations in the $\mathrm{Bal}$ kans is contemplated. To evaluate the extent of genetic variation in the Brenta bears as well as their genetic relationship to Romanian bears, we looked at the sequence variation in mitochondrial DNA from the Italian bears. Nucleic acids were extracted from excremental remains, presumed from their morphology to be of bear origin. All extracts contained large amounts of bacterial DNA.

Two oligonucleotide primers were designed to span an 88-base-pair-long segment of the mitochondrial control region

a, PCR amplifications of bear mitochondrial DNA from three droppings. Lane $M$, size marker ( $\Phi \times 174$ DNA digested with the restriction enzyme HaellI); lanes 1-3, three droppings from the Brenta population; lanes 4 and 5 , extraction and PCR controls, respectively. Upper panel, amplification of a 141-base-pair (bp) fragment (including primers) from the mitochondrial control region. Lower panel, amplification of a 356-bp fragment (including primers) of the chloroplast $r b c L$ gene. $b$, Nucleotide sequences of the 88-bp mitochondrial fragment from three individuals of the Brenta population (Brenta 1, 2 and 3 ). Sequences are compared with the American brown bear ${ }^{2}$ and a brown bear from Romania. Droppings $(0.5 \mathrm{~g})$ were extracted as described in ref. 3, except that the phenol/chloroform and concentration steps were replaced by silica purification ${ }^{4}$. For PCR, we used $25-\mu$ l reaction volumes

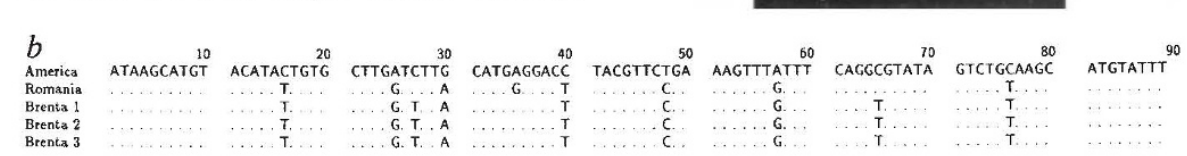

containing BSA ( $\left.2 \mu \mathrm{g} \mathrm{mg}^{-1}\right)$ to overcome inhibition and $10 \mu \mathrm{l}(10 \%)$ extract. Each of $40 \mathrm{PCR}$ cycles consisted of denaturation at $92^{\circ} \mathrm{C}$ for $40 \mathrm{~s}$, annealing at $55^{\circ} \mathrm{C}$ for $60 \mathrm{~s}$ and extension at $72{ }^{\circ} \mathrm{C}$ for $60 \mathrm{~s}$. Primers for the mitochondrial fragment: 5 '-CGTGCATTAATGGCGTGCCCCATGCAT-3' and 5'-TGGTGATCAAGCTCCCGGACTAAGTG-3', and for the rbcL fragment: $5^{\prime}$-ATGTCACCACAAACAGAAACTAAAGCAAGT-3' and 5'-CCAAGACAATGATTGAACAATACTTC-3'. Amplified DNA was electrophoresed on $2.8 \%$ low-melting agarose, the bands were cut out and melted in $100 \mu \mathrm{l}$ water and then reamplified in $50 \mu \mathrm{l}$ using the same conditions, except for a $60^{\circ} \mathrm{C}$ annealing temperature. The products of this second amplification were purified using GeneClean (Dianova) and directly sequenced ${ }^{5}$. representative of this genus, $P$. villosa, is found in the Brenta region. Consequently, the fruits of this plant seem to be a dominant component of the bears' diet during late summer when the droppings were collected. Thus the feeding behaviour of animals can be investigated after amplification by PCR of DNA from their excrement. The collection of droppings may also be a useful way to screen large areas for the presence of rare animals.

\section{Matthias Höss}

Michael Kohn

Svante Pääbo

Institute of Zoology,

Luisenstrasse 14,

University of Munich,

W-8000 Munich 2, Germany

Felix Knauer

Wolfgang Schröder

Munich Wildlife Society,

Linderhof 2,

W-8107 Ettal, Germany

1. Taberlet, P. \& Bouvet, J. Nature 358, 197 (1992).

2. Shields, G. F. \& Kocher, T. D. Evolution 45, 218-221 (1991).

3. Pääbo, S., Gifford, J. A. \& Wilson, A. C. Nucleic Acids Res. 16, 9775-9787 (1988)

4. Boom, R. et al. J. clin. Microbiol. 28, 495-503 (1990) 5. Bachmann, B., Lüke, W. \& Hunsmann, G. Nucleic Acids Res. 18, 1309 (1990).

\section{Evidence for some hot dark matter?}

SIR - The recent detection of the large scale microwave background anisotropy by the cosmic background explorer (COBE) satellite is in remarkable agreement with the predictions based on an inflationary scheme in which the dark matter is a mixture of cold and hot components. We briefly summarize how the COBE measurements, inflation, and the observed large-scale structure all seem to point to a flat Universe in which a fraction $(\sim 20-30$ per cent) of the critical energy density resides in massive $(\sim 3-9 \mathrm{eV})$ relic neutrinos. A crucial test of this scheme comes from the anisotropies expected on smaller $\left(\sim 1^{\circ}-2^{\circ}\right)$ angular scales, which are estimated to lie within reach of current and planned experiments.

A generic prediction of inflation is that the Universe contains a critical density of matter. Primordial nucleosynthesis arguments imply that $<90 \%$ of this matter is nonbaryonic, but inflation does not specify what the dark matter should be. Typically, investigators have assumed the dark matter takes one of two possible forms: cold dark matter (CDM; massive cold particles) or hot dark matter (HDM; relic light neutrinos). Although pure HDM has several well-discussed problems, models with pure CDM provide a basis for structure 